\title{
STRONG PSEUDO-CONVEXITY AND SYMMETRIC DUALITY IN NONLINEAR PROGRAMMING
}

\author{
M. S. MISHRA, S. NANDA AND D. ACHARYA ${ }^{1}$
}

(Received 15 November 1983; revised 25 July 1984)

\begin{abstract}
In this note, the weak duality theorem of symmetric duality in nonlinear programming and some related results are established under weaker (strongly Pseudo-convex/strongly Pseudo-concave) assumptions. These results were obtained by Bazaraa and Goode [1] under (stronger) convex/concave assumptions on the function.
\end{abstract}

\section{Introduction}

We use the following notation and terminology throughout the paper. Let $\psi(x, y)$ be a real-valued twice-differentiable function, defined on an open set in $R^{n+m}$ containing $C_{1} \times C_{2}$, where $C_{1}$ and $C_{2}$ are closed convex cones with non-empty interiors in $R^{n}$ and $R^{m}$ respectively. Let $C_{1}^{*}$ be the polar of $C_{1}$, that is

$$
C_{1}^{*}=\left\{z \cdot x^{t} z \leqslant 0 \text { for each } x \in C_{1} \text { where } x^{t} \text { represents the transpose of } x\right\} \text {. }
$$

$C_{2}^{*}$ is defined similarly. $\nabla_{x} \psi\left(x_{0}, y_{0}\right)$ denotes the gradient vector of $\psi$ with respect to $x$ at the point $\left(x_{0}, y_{0}\right), \nabla_{y} \psi\left(x_{0}, y_{0}\right)$ is defined similarly. $\nabla_{x x} \psi\left(x_{0}, y_{0}\right)$ denotes the matrix (Hessian) of second partial derivative with respect to $x$ evaluated at $\left(x_{0}, y_{0}\right) . \nabla_{x y} \psi\left(x_{0}, y_{0}\right), \nabla_{y x} \psi\left(x_{0}, y_{0}\right)$ and $\nabla_{y y} \psi\left(x_{0}, y_{0}\right)$ are defined similarly.

Definition 1. If $f$ is a scalar-valued differentiable function on a convex set $\Gamma \subset R^{n}$, and $K(x, y)$ is an arbitrary positive scalar function satisfying

$$
K(x, y)\{f(y)-f(x)\} \geqslant(y-x)^{t} \nabla f(x),
$$

\footnotetext{
${ }^{1}$ Indian Institute of Technology, Kharagpur-721302, India.

(c) Copyright Australian Mathematical Society 1985, Serial-fee code 0334-2700/85
} 
then we say that $f$ is strongly Pseudo-convex with respect to $K(x, y)$ (see [2], [5]). If $K(x, y)=1$ then (2) reduces to the definition of convex function.

Definition 2. If $f$ is a scalar-valued differentiable function on a convex set $\Gamma \subset R^{n}$ and $K(x, y)$ is an arbitrary positive scalar function satisfying

$$
K(x, y)\{f(y)-f(x)\} \leqslant(y-x)^{t} \nabla f(x),
$$

then we say that $f$ is strongly Pseudo-concave with respect to $K(x, y)$. If $K(x, y)=1$ then (3) reduces to the definition of concave function.

It may be noted that strong Pseudo-convexity is weaker than convexity and stronger than Pseudo-convexity.

It may be remarked here that strong Pseudo-convexity is not a modification of the usual pseudoconvexity, but rather is a special case of invex, as mentioned by Mond [7]. Thus

$$
f(y)-f(x) \geqslant[h(x, y)]^{\prime} \nabla f(x),
$$

with $h(x, y)=(y-x) / K(x, y)$, shows the invex property.

We say that $\psi$ is strongly Pseudo-convex/strongly Pseudo-concave on $C_{1} \times C_{2}$ if and only if $\psi(\cdot, y)$ is strongly Pseudo-convex with respect to a positive scalar function $K_{1}$ on $C_{1}$ for each given $y \in C_{2}$ and $\psi(x, \cdot)$ is strongly Pseudo-concave with respect to a positive scalar function $K_{2}$ on $C_{2}$ for each given $x \in C_{1}$.

Let us consider a pair of nonlinear programs, as follows.

$P_{0}$ (Primal): Minimize $\quad\left\{f(x, y)=\psi(x, y)-y^{t} \nabla_{y} \psi(x, y)\right\}$ subject to $(x, y) \in C_{1} \times C_{2}, \nabla_{y} \psi(x, y) \in C_{2}^{*}$.

$D_{0}$ (Dual): $\quad$ Maximize $\quad\left\{g(x, y)=\psi(x, y)-x^{t} \nabla_{x} \psi(x, y)\right\}$ subject to $(x, y) \in C_{1} \times C_{2},-\nabla_{x} \psi(x, y) \in C_{1}^{*}$.

For notational convenience, the sets of feasible solutions of $P_{0}$ and $D_{0}$ are denoted by $P$ and $D$ respectively, that is

$$
P=\left\{(x, y) \in C_{1} \times C_{2}: \nabla_{y} \psi(x, y) \in C_{2}^{*}\right\}
$$

and

$$
D=\left\{(x, y) \in C_{1} \times C_{2}:-\nabla_{x} \psi(x, y) \in C_{1}^{*}\right\}
$$

\section{Main results}

TheOREM 1. Let $\psi$ be strongly Pseudo-convex/strongly Pseudo-concave on $C_{1} \times C_{2}$ with respect to scalar-valued functions $K_{1} \geqslant 1$ and $K_{2} \geqslant 1$ respectively. Then

$$
\operatorname{Inf}_{(x, y) \in P} f(x, y) \geqslant \operatorname{Sup}_{(x, y) \in D} g(x, y) .
$$


Proof. Let $(x, y) \in P$ and $(u, v) \in D$. It is sufficient to prove that $f(x, y) \geqslant$ $g(u, v)$.

Since $\psi$ is strongly Pseudo-convex/strongly Pseudo-concave on $C_{1} \times C_{2}$ with respect to the scalar-valued functions $K_{1} \geqslant 1$ and $K_{2} \geqslant 1$ respectively, the following two inequalities hold.

$$
K_{\mathrm{i}}(u, x)\left\{\psi(x, y)-{ }_{v}^{\prime}(u, v)\right\} \geqslant(x-u)^{t} \nabla_{u} \dot{\psi}(u, \ddot{v})
$$

or

$$
\begin{array}{r}
\psi(x, v)-\psi(u, v) \geqslant \frac{(x-u)^{t}}{K_{1}(u, x)} \nabla_{u} \psi(u, v), \\
K_{2}(y, v)\{\psi(x, v)-\psi(x, y)\} \leqslant(v-y)^{t} \nabla_{y} \psi(x, y)
\end{array}
$$

or

$$
\psi(x, v)-\psi(x, y) \leqslant \frac{(v-y)^{t}}{K_{2}(y, v)} \nabla_{y} \psi(x, y) .
$$

By multiplying by -1 in (5) and adding it to (4), we get

$$
\begin{aligned}
0 \geqslant & \psi(u, v)+\frac{(x-u)^{t} \nabla_{u} \psi(u, v)}{K_{1}(u, x)}-\psi(x, y)-\frac{(v-y)^{t} \nabla_{y} \psi(x, y)}{K_{2}(y, v)} \\
= & \psi(u, v)+\frac{x^{t} \nabla_{u} \psi(u, v)}{K_{1}(u, x)}-\frac{u^{t} \nabla_{u} \psi(u, v)}{K_{1}(u, x)} \\
& -\psi(x, y)-\frac{v^{t} \nabla_{y} \psi(x, y)}{K_{2}(y, v)}+\frac{y^{t} \nabla_{y} \psi(x, y)}{K_{2}(y, v)} .
\end{aligned}
$$

Since $u \in C_{1}$ and $-\nabla_{u} \psi(u, v) \in C_{1}^{*} \Rightarrow-u^{t} \nabla_{u} \psi(u, v) \leqslant 0$, by the definition of polar, we have

$$
\frac{-u^{\prime} \nabla_{u} \psi(u, v)}{K_{1}(u, x)} \geqslant-u^{t} \nabla_{u} \psi(u, v) \text { as } K_{1}(u, x) \geqslant 1 .
$$

Similarly $y \in C_{2}$ and $\nabla_{y} \psi(x, y) \in C_{2}^{*} \Rightarrow y^{t} \nabla_{y} \psi(x, y) \leqslant 0$. So we have

$$
\begin{gathered}
\frac{y^{t} \nabla_{y} \psi(x, y)}{K_{2}(y, v)} \geqslant y^{t} \nabla_{y} \psi(x, y) \text { as } K_{2}(y, v) \geqslant 1, \\
\frac{x^{\prime} \nabla_{u} \psi(u, v)}{K_{1}(u, x)} \geqslant 0 \text { as }-x^{t} \nabla_{u} \psi(u, v) \leqslant 0 \text { and } K_{1}(u, x) \geqslant 1, \\
\frac{-v^{t} \nabla_{y} \psi(x, y)}{K_{2}(y, v)} \geqslant 0 \text { as } v^{t} \nabla_{y} \psi(x, y) \leqslant 0 \text { and } K_{2}(y, v) \geqslant 1 .
\end{gathered}
$$


Using (7), (8), (9) and (10) in (6), we get

$$
\begin{aligned}
0 & \geqslant \psi(u, v)-u^{t} \nabla_{u} \psi(u, v)-\left\{\psi(x, y)-y^{t} \nabla_{y} \psi(x, y)\right\} \\
& =g(u, v)-f(x, y) \Rightarrow f(x, y) \geqslant g(u, v) .
\end{aligned}
$$

This completes the proof.

Theorem 1 was motivated by the works of Bazaraa and Goode [1] and Dantzig et al. [4], who proved the same result under stronger assumptions on the cone and the function. In [4], the cone was taken to be non-negative orthant and the function convex/concave. Bazaraa and Goode [1] generalized the results of [4] to arbitrary cones. In Theorem 1 we assume the function to be strongly Pseudo-convex/strongly Pseudo-concave, which is weaker than convex/concave.

It may be noted here that the result does not hold only under Pseudo-convexity/Pseudo-concavity assumptions, and this follows from the following example: Let $n=m=1$. Let $C_{1}=\{x: x \geqslant 0\}, C_{2}=\{y: y \geqslant 0\}$. Let $\psi(x, y)=$ $\exp \left(x-y^{2}\right)$. Then it is easy to check that $\psi$ is Pseudo-convex/Pseudo-concave on $C_{1} \times C_{2}$. But in this case

$$
f(0,2)=9 e^{-4} \leqslant g(0,0)=1,
$$

which contradicts Theorem 1. However, the conclusion of Theorem 1 holds under Pseudo-convexity/Pseudo-concavity, provided we make use of an additional feasibility assumption. This has been discussed in [6].

The following results are also true under weaker assumptions on the function. Since the proofs use ideas similar to those used in [1], we state the theorems without proofs.

THEOREM 2. Suppose that $\left(x_{0}, y_{0}\right)$ solves $P_{0}$, and suppose that $\nabla_{y y} \psi\left(x_{0}, y_{0}\right)$ is negative definite. Then $\left(x_{0}, y_{0}\right) \in D$ and $f\left(x_{0}, y_{0}\right)=g\left(x_{0}, y_{0}\right)$. Further, if $\psi$ is strongly Pseudo-convex/strongly Pseudo-concave with respect to scalar-valued functions $K_{1} \geqslant 1$ and $K_{2} \geqslant 1$, then $\left(x_{0}, y_{0}\right)$ is an optimal solution of problem $D_{0}$.

Theorem 3. Suppose that $\left(x_{0}, y_{0}\right)$ solves $D_{0}$, and $\nabla_{x x} \psi\left(x_{0}, y_{0}\right)$ is positive definite. Then $\left(x_{0}, y_{0}\right) \in P$ and $f\left(x_{0}, y_{0}\right)=g\left(x_{0}, y_{0}\right)=\psi\left(x_{0}, y_{0}\right)$. Further, if $\psi$ is strongly Pseudo-convex/strongly Pseudo-concave with respect to scalar-valued functions $K_{1} \geqslant 1$ and $K_{2} \geqslant 1$, then $\left(x_{0}, y_{0}\right)$ is an optimal solution of problem $P_{0}$. 


\section{Special case}

We now consider a special case of the symmetric dual programs, namely the case when the vector $y$ and the corresponding cone $C_{2}$ are deleted from the formulation. Denoting $\psi(x, y)$ by $f(x)$ and $C_{1}$ by $C$, these two problems arise as special cases of $P_{0}$ and $D_{0}$.

$P_{1}$ (Primal): Minimize $f(x)$ sưbject tó $x \in C$.

$D_{1}$ (Dual): $\quad$ Maximize $f(x)-x^{t} \nabla f(x)$ subject to $x \in C$ and $-\nabla f(x) \in C^{*}$.

Theorem 1 holds, that is $x \in C, u \in C$ with $-\nabla f(u) \in C^{*}$ when $f$ is strongly Pseudo-convex with respect to a scalar function $K \geqslant 1$. To prove this, observe that $f$ is strongly Pseudo-convex with respect to scalar function $K \geqslant 1$. So we have

$$
\begin{aligned}
K(u, x)\{f(x)-f(u)\} & \geqslant(x-u)^{t} \nabla f(u)=x^{t} \nabla f(u)-u^{t} \nabla f(u) \\
& \geqslant-u^{t} \nabla f(u) \text { as } x^{t} \nabla f(u) \geqslant 0,
\end{aligned}
$$

that is

$$
f(x)-f(u) \geqslant \frac{-u^{t} \nabla f(u)}{K(u, x)} \geqslant-u^{t} \nabla f(u) \quad \text { as } K(u, x) \geqslant 1,
$$

that is $f(x) \geqslant f(u)-u^{t} \nabla f(u)$, and this completes the proof.

It may be noted that since $y$ is deleted from the problem, a direct application of Theorem 2 does not hold. However, the theorem is indeed true, that is, if $x_{0}$ solves $P_{1}$ then it solves $D_{1}$. In order to show this we need the following Lemma.

Lemma. Consider the problem: minimize $f(x)$ subject to $x \in C$, where $C$ is a closed convex cone. If $x_{0}$ solves the problem, then $-\nabla f\left(x_{0}\right) \in C^{*}$ and $x_{0}^{t} \nabla f\left(x_{0}\right)=0$. If $f$ is strongly Pseudo-convex with respect to an arbitrary positive scalar function $K$, then conditions are sufficient for $x_{0}$ to solve the problem.

Proof. The first part of the proof is same as that of Lemma ([1], page 7) where no strong Pseudo-convexity is required. The second part of the proof is as follows.

If $x_{0}$ solves the problem then $-\nabla f\left(x_{0}\right) \in C^{*}$ and $x_{0}^{t} \nabla f\left(x_{0}\right)=0$. Now assume that $f$ is strongly Pseudo-convex with respect to an arbitrary positive scalar function $K$, and $x_{0} \in C$ with $-\nabla f\left(x_{0}\right) \in C^{*}$ and $x_{0}^{t} \nabla f\left(x_{0}\right)=0$. Then, for each $x \in C$, we have

$$
\begin{aligned}
K\left(x_{0}, x\right)\left\{f(x)-f\left(x_{0}\right)\right\} & \geqslant\left(x-x_{0}\right)^{t} \nabla f\left(x_{0}\right)=x^{t} \nabla f\left(x_{0}\right)-x_{0}^{t} \nabla f\left(x_{0}\right) \\
& =x^{t} \nabla f\left(x_{0}\right) \quad \text { as } x_{0}^{t} \nabla f\left(x_{0}\right)=0 \\
& \geqslant 0 \quad \text { as } x \in C \text { and }-\nabla f\left(x_{0}\right) \in C^{*} \\
& \Rightarrow f(x)-f\left(x_{0}\right) \geqslant 0 \quad \text { as } K>0 \\
& \Rightarrow f(x) \geqslant f\left(x_{0}\right) .
\end{aligned}
$$

This completes the proof. 
It may be noted that if $x_{0}$ is an optimal solution of the primal problem $P_{1}$ then $-\nabla f\left(x_{0}\right) \in C^{*}, x_{0}$ is indeed a feasible solution of the dual $D_{1}$. In other words the optimality of $P_{1}$ ensures the feasibility of $D_{1}$. The following theorem gives a parallel of Theorem 2 .

THEOREM 4. Suppose that $f$ is strongly Pseudo-convex with respect to a scalar function $K \geqslant 1$, and $x_{0}$ solves the problem $P_{1}$. Then $x_{0}$ solves the problem $D_{1}$.

Proof. Let $x$ be a feasible solution of $D_{1}$, that is $x \in C$ and $-\nabla f(x) \in C^{*}$. Since $x_{0}$ solves the problem $P_{1}$ then by the above lemma $-\nabla f\left(x_{0}\right) \in C^{*}$ and $x_{0}^{t} \nabla f\left(x_{0}\right)=0$. Since $f$ is strongly Pseudo-convex with respect to a scalar function $K \geqslant 1$, we have

$$
\begin{aligned}
K\left(x, x_{0}\right)\left\{f\left(x_{0}\right)-f(x)\right\} & \geqslant\left(x_{0}-x\right)^{t} \nabla f(x)=x_{0}^{t} \nabla f(x)-x^{t} \nabla f(x) \\
& \geqslant-x^{t} \nabla f(x) \quad \text { as } x_{0}^{t} \nabla f(x) \geqslant 1,
\end{aligned}
$$

that is

$$
f\left(x_{0}\right)-f(x) \geqslant \frac{-x^{\prime} \nabla f(u)}{K\left(x, x_{0}\right)} \geqslant-x^{t} \nabla f(x),
$$

as $-x^{t} \nabla f(x) \leqslant 0$ and $K\left(x, x_{0}\right) \geqslant 1$, that is

$$
f\left(x_{0}\right) \geqslant f(x)-x^{\prime} \nabla f(x),
$$

that is

$$
f\left(x_{0}\right)-x_{0}^{t} \nabla f\left(x_{0}\right) \geqslant f(x)-x^{t} \nabla f(x),
$$

as $x_{0}^{t} \nabla f(x)=0$.

This shows that $x_{0}$ solves $D_{1}$. The converse of this theorem can be obtained as a special case of Theorem 3, as long as $\nabla_{x x} f\left(x_{0}\right)$ is positive definite.

\section{Acknowledgment}

This paper was written while the first author was a teacher fellow at I. I. T. Kharagpur. He wishes to thank U. G. C. New Delhi and I. I. T. Kharagpur for providing a teacher fellowship, and D. A. V. College, Titilagarh, for grant of leave of absence. 


\section{References}

[1] M. S. Bazaraa and J. J. Goode, “On symmetric duality in nonlinear programming”, Oper. Res. 21 (1973), 1-9.

[2] Suresh Chandra, "Strong Pseudo-convex programming", Indian J. Pure Appl. Math. 3 (1972), 278-282.

[3] W. S. Dorn, "Duality in quadratic programming”, Quart. Appl. Math. 18 (1960), 155-162.

i4j G. $\bar{B}$. Dantzig, E. Eisenberg, R. W. Cottle, "Symmetric duality in nonlinear programming", Pacific J. Math. 15 (1965), 809-812.

[5] Manjulata, "Strong Pseudo-convex programming in Banach space", Indian J. Pure Appl. Math. (1976), 45-78.

[6] M. S. Mishra, S. Nanda and D. Acharya, "Pseudo-convexity and symmetric duality in nonlinear programming", Report, Department of Mathematics, Indian Institute of Technology, Kharagpur, 1984.

[7] B. Mond, "Generalised convexity in mathematical programming", Bull. Austral. Math. Soc. 27 (1983), 185-202. 